\title{
DETERMINAÇÃO DE PERÓXIDO DE HIDROGÊNIO EM ANTISSÉPTICO BUCAL USANDO UM MICRODISPOSITIVO CONTENDO PARTÍCULAS DE AZUL DA PRÚSSIA
}

\author{
Rodrigo Amorim Bezerra da Silva, Rodrigo Henrique de Oliveira Montes, Rodrigo Alejandro Abarza Munoz e Eduardo \\ Mathias Richter* \\ Instituto de Química, Universidade Federal de Uberlândia, Av. João Naves de Ávila, 2121, 38400-902 Uberlândia - MG, Brasil
}

Recebido em 9/9/10; aceito em 16/12/10; publicado na web em 25/3/11

\begin{abstract}
DETERMINATION OF HYDROGEN PEROXIDE IN ANTISEPTIC MOUTHWASH USING A MICRODEVICE CONTAINING PRUSSIAN BLUE PARTICLES. A simple flow-injection amperometric procedure using a three-electrode-integrated sensor for the determination of $\mathrm{H}_{2} \mathrm{O}_{2}$ in antiseptic mouthwash is reported. This method involves the use of a working composite electrode modified with Prussian Blue (PB) particles that was easily adapted as detector in FIA. The best amperometric response was observed for a composite containing 30\% of graphite modified with PB particles (GAP) and 70\% of pure graphite (GR). The proposed method

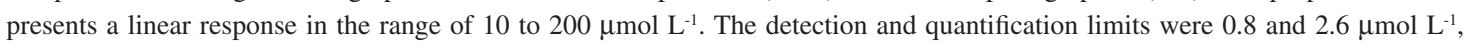
respectively.
\end{abstract}

Keywords: three-electrode-integrated sensor; Prussian Blue particles; hydrogen peroxide.

\section{INTRODUÇÃO}

Sensores modificados com azul da Prússia (AP) insolúvel $\left(\mathrm{Fe}_{4}^{\mathrm{III}}\left[\mathrm{Fe}^{\mathrm{II}}(\mathrm{CN})_{6}\right]_{3}\right)$ têm atraído interesse na área de transdutores eletroquímicos devido à possibilidade de determinação catalítica de $\mathrm{H}_{2} \mathrm{O}_{2}$ em um potencial aplicado relativamente baixo $(0,0 \mathrm{~V}$ vs $\mathrm{Ag} / \mathrm{AgCl} / \mathrm{KClsat}.){ }^{1}$ Neste potencial, uma interferência reduzida de outras espécies é observada, logo, o AP atua como uma "peroxidase artificial". ${ }^{2}$ Peróxido de hidrogênio é uma importante molécula a ser determinada, pois é utilizada em diversas aplicações, como em processos oxidativos avançados para a degradação de poluentes, agente branqueador nas indústrias de papel e celulose, têxteis e produtos de higiene bucal., Além disso, é encontrado no nosso organismo pela ação das enzimas oxidases e na água pela reação da luz solar com substâncias húmicas. ${ }^{3}$ Devido à importância da determinação de $\mathrm{H}_{2} \mathrm{O}_{2}$ e à alta seletividade dos sensores baseados em AP, estes têm atraído grande interesse em diversas áreas como alimentar, ${ }^{5,6}$ ambiental, ${ }^{7}$ forense, ${ }^{8}$ medicinal ${ }^{9}$ e farmacêutica. ${ }^{10}$

A partir do início da década de $90,{ }^{11}$ muitos biossensores têm sido desenvolvidos aliando a alta especificidade das enzimas oxidases (que liberam o $\mathrm{H}_{2} \mathrm{O}_{2}$ como produto de reação) com a seletividade a $\mathrm{H}_{2} \mathrm{O}_{2}$ do AP. Como exemplos de biossensores baseados neste princípio, podemos citar os seletivos a glicose, ${ }^{12,13}$ lactato, ${ }^{14}$ colesterol, ${ }^{15}$, sacarose,${ }^{16}$ dentre outros. ${ }^{7}, 17$ Outras propriedades do AP e seus aná$\log$ os (hexacianoferratos de outros metais), ${ }^{18}$ como a troca iônica ${ }^{19,20}$ e mudanças de $\operatorname{cor}^{21}$ também foram exploradas em determinações analíticas. Revisões com informações detalhadas sobre o assunto podem ser encontradas. ${ }^{12,18,22,23}$

Conforme a literatura, os sensores modificados com AP (e seus análogos) são normalmente preparados de dois modos: deposição eletroquímica em solução com a formação de filmes na superfície de eletrodos sólidos ${ }^{24,25}$ ou a incorporação de partículas de AP em eletrodos de pastas de carbono ${ }^{26}$ ou tintas usadas em eletrodos impressos (screen printed).$^{27}$ Atualmente, uma tendência nos trabalhos envolvendo AP na

*e-mail: emrichter@iqufu.ufu.br construção de sensores é sua síntese em escala nanométrica, merecendo destaque os depositados na superfície de eletrodos pela técnica layer by layer, onde substratos como filmes finos de óxidos mesoporosos (com poros de diâmetro nanométrico ${ }^{28}$ e nanotubos de carbono têm sido utilizados ${ }^{29}$ buscando-se melhora na estabilidade, sensibilidade, reprodutibilidade e robustez dos sensores. Nestes trabalhos, características interessantes podem ser alcançadas devido ao controle das dimensões dos cristais de AP, que dependem da quantidade de camadas depositadas e da grande área superficial das partículas de AP construídas em escala nano. No entanto, é preciso considerar que o custo de produção destes "nano" sensores/biossensores baseados em AP aumentam em função do aprimoramento de sua construção, bem como o número de etapas envolvidas. Neste sentido, uma alternativa bastante viável é a construção de sensores a partir de compósitos fluidos preparados em laboratório ${ }^{30,31}$ modificados com materiais seletivos a certas espécies. Os eletrodos compósitos podem aumentar a resistência mecânica e química (solventes ou meio ácido) do eletrodo, possibilitar o polimento do eletrodo (renovação da superfície) ${ }^{32,33}$ e facilitar a construção de sensores eletroquímicos em escala reduzida.

Neste trabalho apresentamos a caracterização de um microdispositivo que contém os três eletrodos de materiais compósitos imobilizados em uma ponteira de micropipeta, sendo que o eletrodo de trabalho contém o modificador azul da Prússia adsorvido no grafite que compõe o corpo do eletrodo. Este microdispositivo alternativo (3 em 1) pode ser inserido diretamente na tubulação de um sistema de análise por injeção em fluxo (FIA), possibilitando seu uso sem a construção de uma célula eletroquímica para o posicionamento dos eletrodos. Parâmetros do sistema FIA foram otimizados para a determinação de $\mathrm{H}_{2} \mathrm{O}_{2}$ em amostras de antisséptico bucal.

\section{PARTE EXPERIMENTAL}

\section{Reagentes e soluções}

As soluções foram preparadas utilizando água deionizada do sistema de purificação Milli-Q Plus da Millipore (resistividade $>18 \mathrm{M} \Omega \mathrm{cm}$ ). 
Os reagentes eram todos de grau analítico. O compósito fluido de grafite foi construído a partir da mistura de adesivo epóxi Araldite $^{\circledR}$ (Brascola), ciclo-hexanona (Vetec) e grafite (Sigma Aldrich, $\varnothing: 1-2 \mu \mathrm{m}$ ) sob agitação, conforme descrito anteriormente. ${ }^{30,34} \mathrm{O}$ compósito fluido de prata (PC 9045) foi adquirido da empresa Joint Metal Comércio Ltda (Diadema/SP). A modificação do pó de grafite com AP utilizado nos sensores para $\mathrm{H}_{2} \mathrm{O}_{2}$ foi realizada da seguinte maneira: grafite foi adicionado sob agitação (por $10 \mathrm{~min}$ ) a uma mistura equimolar de cloreto férrico e ferricianeto de potássio $\left(0,1 \mathrm{~mol} \mathrm{~L}^{-1}\right)$ em meio de ácido clorídrico $10 \mathrm{mmol} \mathrm{L}^{-1}$. Em seguida, foi separado da solução (por filtração) e colocado em estufa a $100{ }^{\circ} \mathrm{C}$ por $1,5 \mathrm{~h}$ para a ativação das partículas de AP adsorvidas no grafite, ${ }^{26}$ que ocorre pela evaporação das moléculas de água do retículo cristalino do AP. A presença de água na estrutura zeolítica do catalisador diminui a quantidade de sítios disponíveis e prejudica as propriedades condutoras do material. ${ }^{24}$

\section{Construção/otimização do sensor}

O sistema sensor "3 em 1" foi construído pela transferência do compósito fluido de prata e compósito fluido de grafite não modificado em lados diametralmente opostos no lado externo de uma ponteira de micropipeta de polietileno cortada na extremidade inferior (10-100 $\mu \mathrm{L}, \varnothing$ interno $\approx 1,6 \mathrm{~mm}$ ). Internamente foi imobilizado o compósito fluido de grafite modificado com AP. Estes compósitos, após a cura e em contato com fios de cobre atuam como eletrodos de pseudorreferência, auxiliar e trabalho, respectivamente. ${ }^{34} \mathrm{O}$ compósito de grafite contendo AP adsorvido foi testado em diferentes quantidades de grafite modificado no eletrodo e os parâmetros utilizados para a escolha do sensor otimizado foram o excesso superficial $(\Gamma)$, resistência à transferência eletrônica e sensibilidade à $\mathrm{H}_{2} \mathrm{O}_{2}$. Nesta etapa utilizou-se um eletrodo de $\mathrm{Ag} / \mathrm{AgCl} / \mathrm{KCl}$ sat. miniaturizado ${ }^{35}$ e um fio de platina como eletrodos de referência e auxiliar, respectivamente. Antes das medidas, cada sensor foi submetido a 30 voltamogramas cíclicos (de $-0,1$ a $+0,35 \mathrm{~V}$ ) em meio de eletrólito contendo $\mathrm{KCl}$ $0,1 \mathrm{~mol} \mathrm{~L}^{-1}$ para a estabilização do sinal eletroquímico.

\section{Sistema FIA/instrumentação}

Foi utilizado um sistema FIA de linha única e a vazão foi controlada pela pressão constante gerada por uma bomba de aquário em contato com uma coluna d'água. ${ }^{36} \mathrm{Os}$ padrões de $\mathrm{H}_{2} \mathrm{O}_{2}$ e as amostras diluídas foram inseridos no caminho do eletrólito carregador através de um injetor comutador. $\mathrm{O}$ microdispositivo foi diretamente introduzido na extremidade da tubulação do sistema FIA (célula do tipo wall jet).

Os experimentos eletroquímicos foram realizados empregando um potenciostato $\mu$-Autolab III (Eco Chemie) interfaceado a um computador via software GPES 4.9.007. As técnicas eletroquímicas de voltametria cíclica e amperometria a potencial constante foram usadas.

\section{RESULTADOS E DISCUSSÃO}

\section{Otimização do componente grafite do compósito}

A produção do compósito foi realizada conforme publicado anteriormente.$^{30}$ Neste estudo, as proporções entre adesivo epóxi, grafite e ciclo-hexanona foram otimizadas, respectivamente, em 0,04/0,4/0,56 $(\mathrm{m} / \mathrm{m})$. No presente trabalho, os estudos foram direcionados à fração grafite do compósito, onde o objetivo foi identificar a proporção adequada entre grafite modificado com AP (GAP) e grafite puro (GR) para a detecção de $\mathrm{H}_{2} \mathrm{O}_{2}$. A Figura 1 apresenta os resultados obtidos por voltametria cíclica em meio de $\mathrm{KCl} 0,1 \mathrm{~mol} \mathrm{~L}^{-1}$ para eletrodos de compósito produzidos com diferentes proporções entre GAP e GR. A Tabela 1 apresenta os parâmetros voltamétricos obtidos a partir destes experimentos (Figura 1).

Conforme apresentado na Figura 1, em todas as proporções contendo o modificador é possível notar a presença de AP no eletrodo, pelos picos de oxidação do $\mathrm{Fe}(\mathrm{II})$ em aproximadamente $0,2 \mathrm{~V}$ e de redução do $\mathrm{Fe}(\mathrm{III})$, em aproximadamente $0,15 \mathrm{~V}$, característicos do

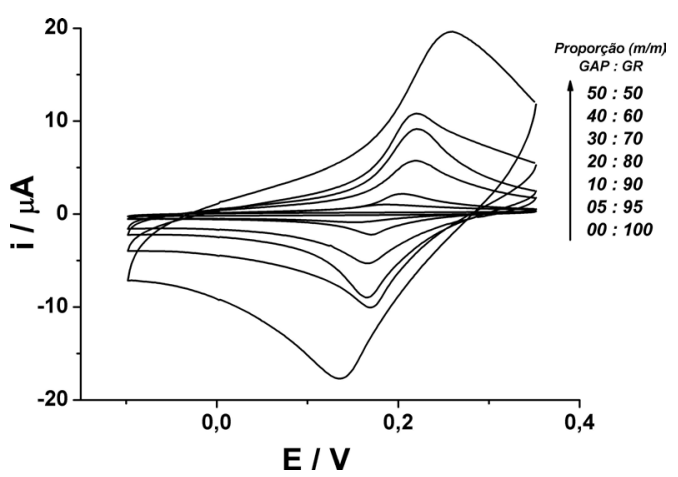

Figura 1. Voltamogramas cíclicos obtidos em $\mathrm{KCl}$ 0,1 $\mathrm{mol} \mathrm{L}^{-1}$ com os eletrodos compósitos construídos com diferentes proporções de GAP:GR. Velocidade de varredura: $20 \mathrm{mV} \mathrm{s}^{-1}$

Tabela 1. Parâmetros voltamétricos para os eletrodos compósito contendo diferentes proporções de grafite modificado com azul da Prússia (GAP) e grafite puro (GR) com os respectivos excessos superficiais $(\Gamma)$ de AP no eletrodo

\begin{tabular}{|c|c|c|c|c|c|c|}
\hline & \multicolumn{6}{|c|}{ Proporções GAP:GR } \\
\hline & $5: 95$ & 10:90 & $20: 80$ & $30: 70$ & $40: 60$ & $50: 50$ \\
\hline Epc $(\mathrm{mV})$ & 154 & 170 & 168 & 166 & 170 & 136 \\
\hline Epa $(\mathrm{mV})$ & 186 & 206 & 218 & 220 & 230 & 260 \\
\hline$\Delta \mathrm{Ep}(\mathrm{mV})$ & 32 & 36 & 50 & 54 & 60 & 124 \\
\hline $\operatorname{Ipc}(\mu \mathrm{A})$ & $-0,88$ & $-2,21$ & $-5,30$ & $-8,98$ & $-10,07$ & $-17,70$ \\
\hline Ipa $(\mu \mathrm{A})$ & 1,03 & 2,18 & 5,73 & 9,15 & 10,80 & 19,64 \\
\hline$\Delta \mathrm{Ip}(\mu \mathrm{A})$ & 1,92 & 4,39 & 11,03 & 18,13 & 20,87 & 37,34 \\
\hline | Ipa / Ipc | & 1,17 & 0,99 & 1,08 & 1,02 & 1,07 & 1,11 \\
\hline $\mathrm{Q}(\mu \mathrm{C})^{*}$ & 0,18 & 0,30 & 0,88 & 1,34 & 1,82 & 3,63 \\
\hline$\Gamma\left(\mathrm{nmol} \mathrm{cm}^{-2}\right)^{* *}$ & 1,16 & 1,93 & 5,67 & 8,64 & 11,73 & 23,40 \\
\hline
\end{tabular}

*Cargas obtidas pelo cálculo das integrais dos voltamogramas cíclicos da Figura 1. ** $\Gamma=\mathrm{Q} / \mathrm{nFA}$; Q: carga (C); n: número de elétrons envolvidos no par redox (4); F: constante de Faraday $\left(96.485 \mathrm{C} \mathrm{mol}^{-1}\right)$; A: área geométrica do eletrodo $\left(0,0201 \mathrm{~cm}^{2}\right)$. 
azul da Prússia e branco da Prússia, respectivamente. No material não modificado (proporção 00:100; Figura 1), estes picos não estão presentes. Conforme pode ser observado (Figura 1 e Tabela 1), um aumento nos valores de $\Delta \mathrm{Ip}$ ocorre em eletrodos com proporções maiores de GAP, o que é consequência de uma maior quantidade de AP no corpo do eletrodo. Adicionalmente, existe uma variação nos valores de Ep e $\Delta \mathrm{E}_{\mathrm{p}}$ em função da quantidade de AP na mistura do grafite. De acordo com a Tabela 1, quanto maior a quantidade de GAP presente no eletrodo, maiores são as separações dos potenciais de pico anódico e catódico. Este aumento de $\Delta$ Ep é causado por um efeito de queda ôhmica. Estas limitações identificadas em sensores contendo altas frações de AP são semelhantes aos reportados na literatura quando eletrodos impressos ${ }^{27}$ ou pasta de carbono ${ }^{37}$ foram utilizados. De acordo com estes trabalhos, as partículas de GAP são menos condutoras do que as partículas de GR, logo a resistência aumenta com o aumento da porcentagem de GAP. Os eletrodos modificados com AP nas diversas proporções também foram testados em função da resposta a $\mathrm{H}_{2} \mathrm{O}_{2}$ por amperometria com a aplicação de um potencial constante de $0,0 \mathrm{~V}$ vs $\mathrm{Ag} / \mathrm{AgCl} / \mathrm{KCl}$ sat. As curvas de calibração para estes experimentos são apresentados na Figura 2A.
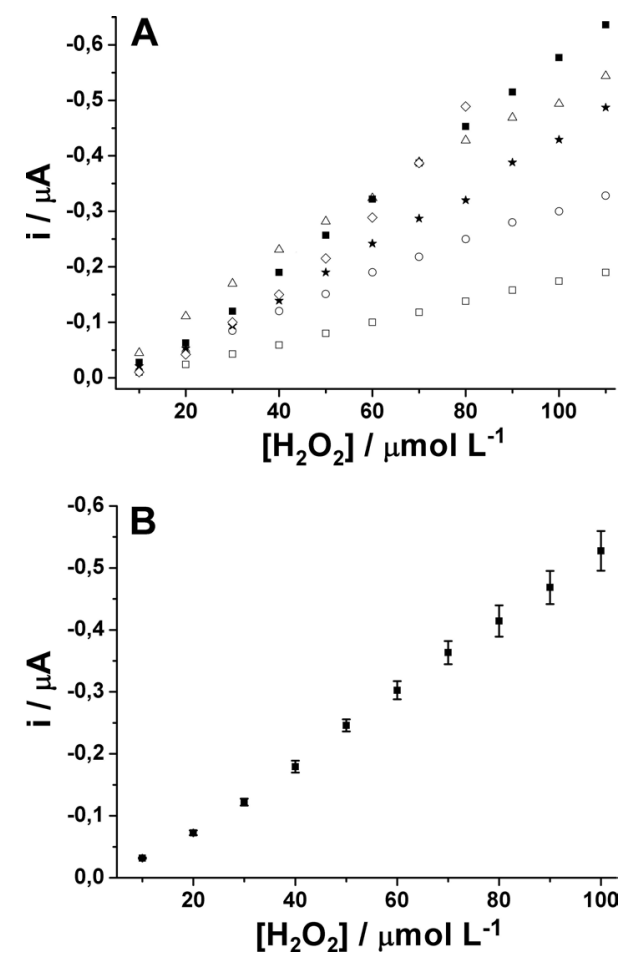

Figura 2. (A) Curvas de calibração obtidas para eletrodos construídos em diferentes proporções de GAP:GR ( $\square$ - 05:95; О - 10:90; $\triangle$ - 20:80; - 30:70; $\star$ - 40:60; $\diamond-50: 50) ;(B)$ curva de calibração média $( \pm D P)$ de 5 eletrodos distintos de composição 30:70 (GAP:GR). Analito: $\mathrm{H}_{2} \mathrm{O}_{2}$; potencial aplicado: 0,0 V; eletrólito: $\mathrm{KCl} 0,1 \mathrm{~mol} \mathrm{~L}^{-1}$

Considerando os resultados apresentados na Figura 2A, a intensidade de resposta dos eletrodos de composição 05/95, 10/90, 20/80 e 30/70 (GAP/AP, respectivamente) é proporcional ao aumento da quantidade de AP na superfície do eletrodo. Em proporções de GAP maiores (40/60 e 50/50), o efeito de queda ôhmica prevalece sobre o excesso de AP na superfície do eletrodo, deixando o eletrodo mais resistivo (Figura 1 e Tabela 1), mais instável e com menor razão sinal/ruído (resultados não apresentados). O comportamento resistivo é altamente dependente da fração de GAP no compósito, porque o AP está presente no corpo do eletrodo. Em eletrodos de filmes de AP (automontados ou eletrodepositados), este fenômeno é menos pronunciado, pois o modificador somente está presente na superfície do eletrodo. Em função disto, o eletrodo de composição intermediária (30/70 em GAP/GR) apresentou o melhor desempenho considerando simultaneamente características como resistência à transferência eletrônica, sensibilidade e estabilidade. Nesta proporção de GAP:GR, o teor calculado de AP no eletrodo compósito é de $2,15 \%(\mathrm{~m} / \mathrm{m})$. A Figura 2B mostra as respostas obtidas para 5 eletrodos diferentes construídos com a proporção 30:70 em função de concentrações crescentes de $\mathrm{H}_{2} \mathrm{O}_{2}$. Devido aos baixos desvios padrões calculados (Figura 2B), nota-se uma ótima reprodutibilidade intereletrodo, o que pode ser atribuído a uma alta homogeneidade do compósito de grafite fluido utilizado na construção destes sensores, pois a boa fluidez do material permite a homogeneização do compósito usando-se um agitador magnético.

Conforme trabalho anterior, ${ }^{30}$ uma das vantagens da preparação de compósitos fluidos é a possibilidade de utilização da mesma mistura (adesivo epóxi, grafite e ciclo-hexanona) durante 1 mês, pois o material permanece fluido neste intervalo de tempo (frasco hermeticamente fechado). A cura somente ocorre com a fração de material usada na construção de um sensor. No entanto, esta longevidade não é válida quando o modificador AP faz parte da mistura (compósito fluido). Experimentos voltamétricos (não apresentados) realizados com eletrodos preparados a partir de um compósito fluido modificado (adesivo epóxi + grafite + ciclo-hexanona $+\mathrm{AP}$ ) preparado a mais de 1 semana apresentaram picos de oxidação e/ou redução anômalos, devido à provável dessorção e/ou decomposição do mediador no compósito fluido. No entanto, quando o AP é adicionado à mistura e os sensores são construídos no mesmo dia (cura do compósito), o sensor modificado permanece funcional por um período superior a 6 meses (tempo avaliado), desde que o eletrodo compósito seja utilizado no intervalo da sua faixa de trabalho. Segundo estudos realizados, a atividade catalítica permanece constante neste período, desde que a renovação da superfície seja efetuada com suave polimento (lixa d'água de granulação 1200) antes de seu uso, pois o compósito atua como um reservatório de AP.

Após os estudos de otimização e definição da proporção de grafite modificado com AP a ser usado no eletrodo de trabalho, o dispositivo foi adaptado a um sistema FIA de linha única para quantificação de $\mathrm{H}_{2} \mathrm{O}_{2}$. Para facilitar a adaptação do dispositivo ao sistema FIA (inserção diretamente na tubulação), os três eletrodos que compõem a célula amperométrica foram posicionados na extremidade de uma ponteira de micropipeta $(10-100 \mu \mathrm{L}) \cdot{ }^{38}$ Neste dispositivo, o eletrodo de referência $\mathrm{Ag} / \mathrm{AgCl} / \mathrm{KClsat}$. foi substituído por um pseudorreferência (compósito de prata). Como o pseudorreferência possui um deslocamento de potencial de $-100 \mathrm{mV}$ em relação ao $\mathrm{Ag} / \mathrm{AgCl} /$ KClsat., o potencial de $0,0 \mathrm{~V}$ foi substituído por $-0,1 \mathrm{~V} \cdot{ }^{38} \mathrm{~A}$ Figura 3 mostra os resultados obtidos em função da variação dos parâmetros vazão e volume de injeção.

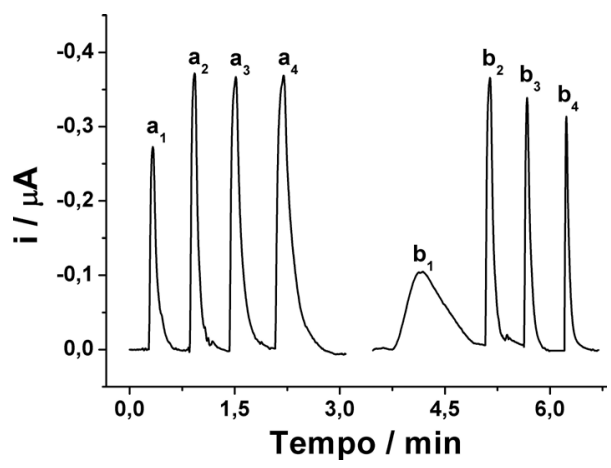

Figura 3. Amperogramas obtidos para injeção de $50 \mu \mathrm{mol} \mathrm{L} \mathrm{L}^{-1}$ de $\mathrm{H}_{2} \mathrm{O}_{2} \mathrm{em}$ função do: (a) volume injetado $\left(a_{1}=65 ; a_{2}=100 ; a_{3}=165 ; a_{4}=230 \mu \mathrm{L}\right)$; (b) vazão $\left(b_{1}=0,4 ; b_{2}=1,0 ; b_{3}=2,0\right.$ e $\left.b_{4}=3,0 \mathrm{~mL} \mathrm{~min}{ }^{-1}\right)$. Sensor utilizado: composição 30:70. Eletrólito: $\mathrm{KCl}$ 0,1 mol L-1. Potencial aplicado: - 0,1 V 
Como pode ser visto na Figura 3, a injeção de volumes superiores a $100 \mu \mathrm{L}\left(\mathrm{a}_{2}\right)$ não gera aumento no sinal amperométrico, somente alargamento do pico e, consequentemente, menor frequência analítica. Em relação à vazão, observa-se sinal máximo de corrente de pico em 1,0 $\mathrm{mL} \mathrm{min}^{-1}$. Vazões inferiores fornecem picos pouco intensos e largos e vazões superiores resultam em picos mais estreitos, porém, menores, o que é consequência de uma limitação cinética do mediador AP (redução eletrocatalítica do $\mathrm{H}_{2} \mathrm{O}_{2}$ em menor extensão). Logo, o volume injetado de $100 \mu \mathrm{L}$ a uma vazão de $1,0 \mathrm{~mL} \mathrm{~min}^{-1}$ fornece a melhor condição em relação à frequência analítica e sensibilidade. Nestes estudos, o percurso analítico foi de $20 \mathrm{~cm}(\varnothing$ interno $=1,0$ $\mathrm{mm}$ ). Nesta condição, o eletrodo de proporção 30:70 foi submetido a sucessivas injeções de $\mathrm{H}_{2} \mathrm{O}_{2} 75,0 \mu \mathrm{mol} \mathrm{L} \mathrm{L}^{-1}$ e um baixo desvio padrão relativo foi calculado $(0,7 \% ; \mathrm{n}=10)$.

A Figura 4 apresenta os resultados obtidos para injeções de soluções com concentrações crescentes e decrescentes de $\mathrm{H}_{2} \mathrm{O}_{2}$ e duas amostras de antisséptico bucal (a e b) usando as condições otimizadas. As duas curvas analíticas obtidas para o sentido crescente e decrescente apresentaram linearidade no intervalo de concentração avaliado (entre 10 e $200 \mu \mathrm{mol} \mathrm{L}^{-1}$ ), sendo descritas pelas respectivas equações: $\mathrm{I}(\mu \mathrm{A})=-0,01345-0,00546\left[\mathrm{H}_{2} \mathrm{O}_{2}\right]\left(\mu \mathrm{mol} \mathrm{L}{ }^{-1}\right)$ e I $(\mu \mathrm{A})$ $=-0,02017-0,00540\left[\mathrm{H}_{2} \mathrm{O}_{2}\right]\left(\mu \mathrm{mol} \mathrm{L} \mathrm{L}^{-1}\right), \mathrm{com} \mathrm{r}=0,999$ para ambas as equações. Os limites de detecção e quantificação (3 e 10 vezes o desvio padrão do branco, respectivamente) $)^{39}$ foram calculados em 0,8 e 2,6 $\mu \mathrm{mol} \mathrm{L} \mathrm{L}^{-1}$. Nestas condições, a frequência analítica obtida foi de aproximadamente 100 injeções/h. A Tabela 2 apresenta os resultados obtidos na determinação de $\mathrm{H}_{2} \mathrm{O}_{2}$ em duas amostras de antisséptico bucal, empregando o método proposto e o método iodométrico. Segundo o teste-t pareado $\left(\mathrm{t}_{\exp }=3,28 v s \mathrm{t}_{\text {tab }}=4,30\right)$, os resultados obtidos em relação ao teor de $\mathrm{H}_{2} \mathrm{O}_{2}$ nas amostras apresentaram boa
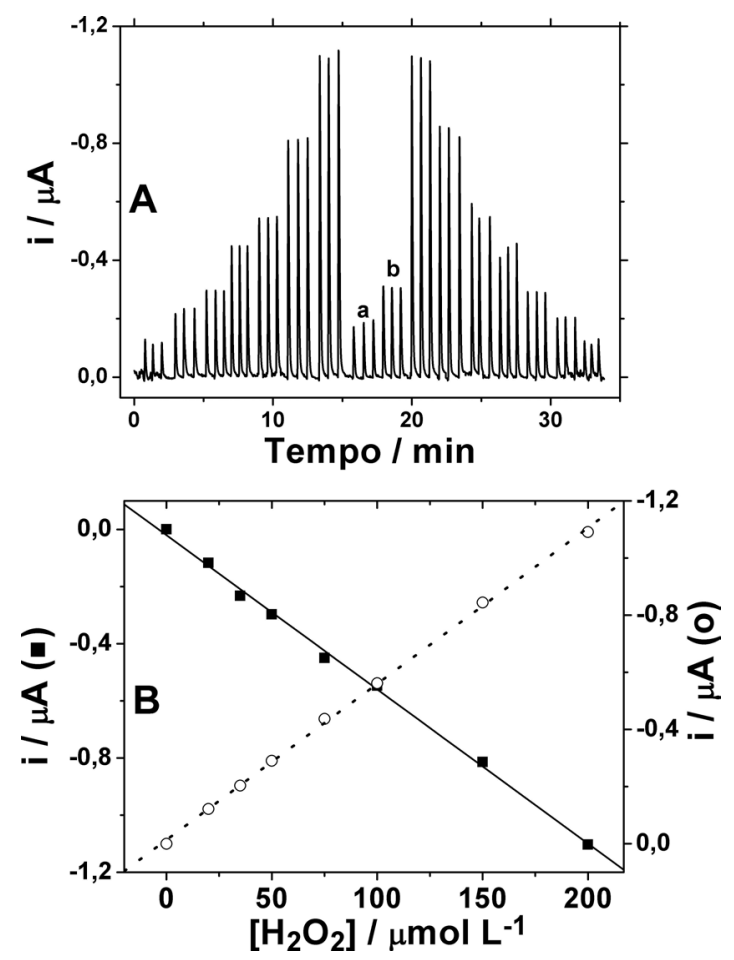

Figura 4. (A) Amperograma obtido para injeção $(n=3)$ de soluções padrão de $\mathrm{H}_{2} \mathrm{O}_{2}$ no sentido crescente de concentrações (20, 35, 50, 75, 100, 150 e $200 \mu \mathrm{mol} \mathrm{L^{-1 }}$ ), duas amostras de antisséptico bucal (a e b) e dos padrões no sentido decrescente. (B) Respectivas curvas de calibração obtidas no sentido crescente (ם) e decrescente (O) dos padrões de $\mathrm{H}_{2} \mathrm{O}_{2}$. Eletrólito: tampão fosfato 0,05 mol $\mathrm{L}^{-1}$ e $\mathrm{KCl} 0,1 \mathrm{~mol} \mathrm{~L}^{-1}(\mathrm{pH} \mathrm{7,2)}$. Outras condições, conforme Figura 3
Tabela 2. Comparação dos resultados obtidos pelo método proposto (amperométrico) e por titulação iodométrica na determinação de $\mathrm{H}_{2} \mathrm{O}_{2}$ em amostras de antisséptico bucal

\begin{tabular}{|c|c|c|c|}
\hline \multirow[b]{2}{*}{ Amostras } & \multicolumn{3}{|c|}{ Concentração de $\mathrm{H}_{2} \mathrm{O}_{2} /(\%)$} \\
\hline & FIA/Amperometria ${ }^{\mathrm{a}}$ & Iodometria $^{\mathrm{a}}$ & Erro relativo $/(\%)$ \\
\hline $\mathbf{a}$ & $1,19 \pm 0,04$ & $1,12 \pm 0,03$ & 6,1 \\
\hline b & $1,94 \pm 0,05$ & $1,86 \pm 0,04$ & 4,1 \\
\hline
\end{tabular}

${ }^{\mathrm{a}}$ Média de 3 medidas. ${ }^{\mathrm{b}}$ Erro relativo $=100 \mathrm{x}$ (valor amperométrico - valor iodométrico) / valor iodométrico.

concordância entre o método proposto e o método iodométrico a um nível de confiança de $95 \%(n=3)$.

A Tabela 3 apresenta os resultados obtidos nos estudos de adição e recuperação. Três diferentes concentrações de $\mathrm{H}_{2} \mathrm{O}_{2}$ foram adicionadas em duas amostras de antisséptico bucal. Como pode ser observado, as recuperações ficaram próximas a $100 \%$, evidenciando que não há interferência significativa da matriz da amostra.

Tabela 3. Resultados obtidos nos estudos de recuperação para determinação de $\mathrm{H}_{2} \mathrm{O}_{2}$ em amostras de antisséptico bucal usando o método proposto

\begin{tabular}{cccc}
\hline Amostra & $\begin{array}{c}{\left[\mathrm{H}_{2} \mathrm{O}_{2}\right] \text { adicionada }} \\
\left(\mu \mathrm{mol} \mathrm{L} \mathrm{L}^{-1}\right)\end{array}$ & $\begin{array}{c}{\left[\mathrm{H}_{2} \mathrm{O}_{2}\right] \text { encontrada }} \\
\left.(\mu \mathrm{mol} \mathrm{L})^{-1}\right)\end{array}$ & $\begin{array}{c}\text { Recuperação } \\
(\%)\end{array}$ \\
\hline a & 0 & 52,7 & -- \\
& 50 & 100,4 & 97,7 \\
& 100 & 150,1 & 98,2 \\
b & 150 & 194,0 & 95,7 \\
& 0 & 83,7 & -- \\
& 100 & 138,3 & 104,0 \\
& 150 & 181,2 & 98,6 \\
\hline
\end{tabular}

*Solução diluída uma vez antes da análise.

\section{CONCLUSÕES}

$\mathrm{O}$ método proposto para quantificação de $\mathrm{H}_{2} \mathrm{O}_{2}$ em amostras de antisséptico bucal apresenta as seguintes vantagens: simples diluição como etapa única de pré-tratamento das amostras; nenhuma necessidade de adição de reagentes, pois a redução catalítica do $\mathrm{H}_{2} \mathrm{O}_{2}$ ocorre na superfície do eletrodo modificado com AP; alta frequência analítica proporcionada pelo sistema FIA e consumo reduzido de amostras e reagentes. Além disto, estes microdispositivos dispensam o uso de eletrodos de referência ou auxiliar externos, pois o dispositivo contém os três eletrodos posicionados na extremidade da ponteira, o que permite sua adaptação diretamente na extremidade da tubulação do sistema FIA. A presença de AP no corpo do eletrodo de trabalho permite fácil recuperação da atividade catalítica do mediador através de um simples polimento da superfície do eletrodo. Isto não é possível quando o filme de AP é depositado sobre um eletrodo ou mesmo quando o AP é imobilizado em eletrodos impressos. Finalmente, os sensores apresentados no trabalho são de fácil construção e de baixo custo, logo são passíveis de serem construídos em qualquer laboratório.

\section{AGRADECIMENTOS}

Ao IQ-UFU, ao programa multi-institucional de doutorado UFU/ UFG/UFMS e à CAPES, FAPEMIG e ao CNPq pelo apoio financeiro. R. A. B. da Silva agradece ao CNPq pela bolsa de doutorado concedida (141972/2009-2). 


\section{REFERÊNCIAS}

1. Itaya, K.; Akahoshi, H.; Toshima, S.; J. Electrochem. Soc. 1982, 129, 1498.

2. Karyakin, A. A.; Karyakina, E. E.; Gorton, L.; J. Electroanal. Chem. 1998, 456, 97.

3. de Mattos, I. L.; Shiraishi, K. A.; Braz, A. D.; Fernandes, J. R.; Quim. Nova 2003, 26, 373.

4. Vidal, I. G.; Dias, I. L. T.; de Oliveira, G.; Lanza, M. R. D.; Sotomayor, M. D. T.; Anal. Lett. 2009, 42, 352.

5. de Mattos, I. L.; Areias, M. C. D.; Talanta 2005, 66, 1281.

6. Adhoum, N.; Monser, L.; Sens. Actuators, B 2008, 133, 588.

7. Ivanov, A.; Evtugyn, G.; Budnikov, H.; Ricci, F.; Moscone, D.; Palleschi, G.; Anal. Bioanal. Chem. 2003, 377, 624.

8. Munoz, R. A. A.; Lu, D. L.; Cagan, A.; Wang, J.; Analyst 2007, 132, 560.

9. Wang, Y. T.; Zhu, J. Z.; Zhu, R. J.; Zhu, Z. Q.; Lai, Z. S.; Chen, Z. Y.; Meas. Sci. Technol. 2003, 14, 831 .

10. Derwinska, K.; Miecznikowski, K.; Koncki, R.; Kulesza, P. J.; Glab, S.; Malik, M. A.; Electroanalysis 2003, 15, 1843.

11. Karyakin, A. A.; Gitelmacher, O. V.; Karyakina, E. E.; Anal. Chem. 1995, 67, 2419.

12. Ricci, F.; Moscone, D.; Tuta, C. S.; Palleschi, G.; Amine, A.; Poscia, A. Valgimigli, F.; Messeri, D.; Biosens. Bioelectron. 2005, 20, 1993.

13. Ahmadalinezhad, A.; Kafi, A. K. M.; Chen, A. C.; Electrochem. Commun. 2009, 11, 2048.

14. Lin, Y. Q.; Liu, K.; Yu, P.; Xiang, L.; Li, X. C.; Mao, L. Q.; Anal. Chem. 2007, 79, 9577.

15. Li, J. P.; Peng, T. Z.; Peng, Y. Q.; Electroanalysis 2003, 15, 1031.

16. Haghighi, B.; Varma, S.; Alizadeh, F. M.; Yigzaw, Y.; Gorton, L.; Talanta 2004, 64, 3 .

17. Varma, S.; Yigzaw, Y.; Gorton, L.; Anal. Chim. Acta 2006, 556, 319.

18. de Mattos, I. L.; Gorton, L.; Quim. Nova 2001, 24, 200.
19. Castilho, M. D.; Stradiotto, N. R.; Talanta 2008, 74, 1630.

20. Eftekhari, A.; Anal. Lett. 2003, 36, 263.

21. Lenarczuk, T.; Glab, S.; Koncki, R.; J. Pharm. Biomed. Anal. 2001, 26, 163.

22. Karyakin, A. A.; Karyakina, E. E.; Russ. Chem. Bull. 2001, 50, 1811.

23. Koncki, R.; Crit. Rev. Anal. Chem. 2002, 32, 79.

24. de Mattos, I. L.; Gorton, L.; Ruzgas, T.; Karyakin, A. A.; Anal. Sci. 2000, 16, 795

25. Paixao, T.; Bertotti, M.; Sens. Actuators, B 2009, 137, 266

26. Moscone, D.; D’Ottavi, D.; Compagnone, D.; Palleschi, G.; Amine, A.; Anal. Chem. 2001, 73, 2529.

27. O’Halloran, M. P.; Pravda, M.; Guilbault, G. G.; Talanta 2001, 55, 605.

28. Gaitan, M.; Goncales, V. R.; Soler-Illia, G.; Barald, L. M.; de Torresi, S. I. C.; Biosens. Bioelectron. 2010, 26, 890.

29. Li, J.; Yao, Y. L.; Shiu, K. K.; Anal. Sci. 2010, 26, 431.

30. da Silva, R. A. B.; Rabelo, A. C.; Bottecchia, O. L.; Munoz, R. A. A.; Richter, E. M.; Quim. Nova 2010, 33, 1398.

31. Barsan, M. M.; Pinto, E. M.; Florescu, M.; Brett, C. M. A.; Anal. Chim. Acta 2009, 635, 71.

32. Ramirez-Garcia, S.; Cespedes, F.; Alegret, S.; Electroanalysis 2001, 13, 529.

33. Calixto, C. M. F.; Cervini, P.; Cavalheiro, E. T. G.; Quim. Nova 2008, 31, 2194.

34. da Silva, R. A. B.; de Almeida, E. G. N.; Rabelo, A. C.; da Silva, A. T. C.; Ferreira, L. F.; Richter, E. M.; J. Braz. Chem. Soc. 2009, 20, 1235.

35. Pedrotti, J. J.; Angnes, L.; Gutz, I. G. R.; Electroanalysis 1996, 8, 673.

36. dos Santos, W. T. P.; Ceolin, M. P.; de Albuquerque, Y. D. T.; Richter, E. M.; Quim. Nova 2007, 30, 1754.

37. Ricci, F.; Goncalves, C.; Amine, A.; Gorton, L.; Palleschi, G.; Moscone, D.; Electroanalysis 2003, 15, 1204.

38. da Silva, R. A. B.; Rabelo, A. C.; Munoz, R. A. A.; Richter, E. M.; Electroanalysis 2010, 22, 2167.

39. Miller, J. C.; Miller, J. N. Statistics for Analytical Chemistry; Harwood: Chichester, 1992. 